\title{
Thermodynamic and Conformational Properties of Styrene-Methyl Methacrylate Block Copolymers in Dilute Solution. V. Light-Scattering Analysis of Conformational Anomalies in $p$-Xylene Solution
}

\author{
Takeshi Tanaka, Tadao Kotaka, and Hiroshi Inagaki \\ Institute for Chemical Research, Kyoto University \\ Uji, Kyoto-fu 611, Japan.
}

(Received August 30, 1971)

\begin{abstract}
Light-scattering studies were made in order to examine conformational anomalies exhibited by solutions in $p$-xylene $(p \mathrm{XY})$ of nearly equimolar AB-diblock and BAB-triblock copolymers, wherein A denotes polystyrene (PST) and B poly(methyl methacrylate) (PMMA). Particle-scattering function $P(\theta)$ was calculated on two simplified models: one is a micelle consisting of $f$ molecules of AB-diblock type, of which B-subchains constitute the core (star-shape model); and the other represents a BAB-triblock molecule, in which two side B-subchains undergo intramolecular association, forming a droplet, and the conformation of A-subchain is restricted by this droplet (arc-shape mode1). The calculated $P(\theta)$ functions were compared with the experimental data obtained in $p \mathrm{XY}$ solution at $30^{\circ} \mathrm{C}$ from $\mathrm{AB}$-diblock and $\mathrm{BAB}$-triblock samples. It was found that the star-shape model fits closely the behavior of the AB-diblock system; whilst the arc-shape model appears to give a reasonable description of the behavior of the BAB-triblock system (although the results are not completely conclusive).

KEY WORDS Light-scattering / Particle-Scattering Function / ABDiblock Copolymer / BAB-Triblock Copolymer / Conformational Anomaly / Intramolecular Phase Separation / Micelle Formation / Intramolecular Association / Star-Shape Model / Arc-Shape Model /
\end{abstract}

In the previous papers ${ }^{1-5}$ of this series the dilute solution behavior of nearly equimolar styrene (ST) and methyl methacrylate (MMA) block copolymers was explored in some detail. The materials studied were those of AB-diblock and BAB-triblock type, wherein A denotes polystyrene (PST) and B denotes poly(methyl methacrylate) (PMMA). In the comparison of their behavior the following two points were particularly noticed. ${ }^{4,5}$ The one was that in certain selective solvents $\left(e . g ., p\right.$-xylene at $\left.30^{\circ} \mathrm{C}\right)$ having unfavorable solvency toward PMMA, the intrinsic viscosities $[\gamma]$ of some BAB-triblock samples were found to become smaller than those of their precursor PST. The other was that, in such solvents, the AB-diblock samples

* Part of this work was presented at the 20th Polymer Symposium of the Society of Polymer Science, Japan, held in Tokyo, October, 1971. often underwent stable micelle formation by intermolecular association, whilst under the same condition the BAB-triblock samples did not form micelles but remained in the state of molecular dispersion.

To interpret such behavior, a concept of the so-called intramolecular phase separation was introduced. In poor or nonsolvents toward homopolymer B, the B-subchain(s) of an individual block copolymer molecule might collapse; this phenomenon and the poor compatibility of the parent homopolymer pair in the solvent would induce the intramolecular phase separation. ${ }^{4}$ Then AB-diblock molecules, each having one soluble A-subchain and one collapsing B-subchain, would undergo intermolecular association forming stable micelles. In such a micelle the collapsing B-subchains would accumulate in the core and the soluble A subchains would protect the micelle from further aggregation. On the 
other hand the situation appeared to be somewhat different for BAB-triblock molecules, each of which possesses a soluble A-subchain flanked by two collapsing B-subchains. Evidences from sedimentation equilibrium and light-scattering studies had revealed that the BAB-triblock molecules did not undergo intermolecular association in such solvents. ${ }^{5}$ Hence the $[\eta]$ anomalies must have resulted from conformational anomalies of the individual triblock molecules. A possible explanation was that the two collapsing B-subchains within a BABtriblock molecule might undergo intramolecular association, and the central A-subchain might be forced to assume a semi-circular conformation surrounding the droplet formed by the intramolecular association of the two B-subchains. Such a conformation would effectively prevent the further intermolecular association of the triblock molecules. This hypothetical conformation is admittedly too crude, but explains at least qualitatively the differences found between the behavior of AB-diblock and BABtriblock molecules in $p$-xylene ( $p \mathrm{XY})$ solutions. ${ }^{5}$ Of course there are many other possible explanations that might apply to these anomalies as well. For example, the different behavior might be simply due to the difference in the molecular weight of the A-subchain relative to that of the B-subchain: namely, in the BABtriblock molecule the A-subchain is about twice as long as the individual B-subchain, whilst in the AB-diblock molecule they are of nearly identical length.

A useful method for examining these possible anomalies is observation of the angular distribution of Rayleigh scattering from block copolymer solutions. To this end an examination was attempted of the light-scattering behavior of the nearly equimolar PST-PMMA diblock and PMMA-PST-PMMA triblock copolymers in $p \mathrm{XY}$ solutions. The advantage of using $p X Y$ as a salvent is two-fold. In the first place, $p \mathrm{XY}$ is a good solvent for PST but a $\theta$ solvent toward PMMA $\left(\theta=\right.$ about $\left.40^{\circ} \mathrm{C}\right)$ and has little power to induce compatibility between PST and PMMA pair; ${ }^{4}$ and we already have found the anomalies as described above. ${ }^{4,5}$ Secondly the specific refractive increment of PMMA in $p X Y$ has been found to be nearly zero, i.e., the value is $0.007(\mathrm{~m} l / \mathrm{g})$ at $30^{\circ} \mathrm{C}$ for $436 \mathrm{~m} \mu$ wave-length light. The value is to be compared with 0.114 for PST under the same conditions. Therefore one can observe the light scattering mainly from the PST-subchains alone, and this feature would make the analysis much simpler.

The purpose of this work is to calculate the Debye $^{6}$ particle-scattering function $P(\theta)$ for a few models which would be appropriate in describing the features of the PST and PMMA block copolymer solutions as described above. The results will be compared with experimentally observable $\boldsymbol{P}(\theta)$ functions for the PST-PMMA diblock and PMMA-PST-PMMA triblock copolymers in $\mathrm{pXY}$ solutions.

\section{CALCULATION OF PARTICLE-SCATTERING FUNCTION $\boldsymbol{P}(\theta)$}

\section{Statistical Models}

For the calculation of $P(\theta)$, we employ two models as illustrated in Figures 1a and 1b: the one shown in Figure 1a represents a micelle consisting of AB-diblock copolymers and the other in Figure $1 \mathrm{~b}$ represents a BAB-triblock copolymer molecule. It is assumed that Bsubchains are less easily visible in the given solvent, which in addition is a poor solvent toward homopolymer B. For the AB-diblock copolymer system, it is assumed that $f$ molecules, each consisting of $N_{A}$ freely jointed segments of type A and $N_{B}$ segments of type B, form a star-shape micelle with $f$ branches and a spherical core of radius $R_{1}=L_{1} / 2$ : the B-segments constitute the core and the first segment of each A-branch is localized on the surface of the core (see Figure 1a). For the BAB-triblock copolymer system it is assumed that the two B-subchains, each consisting of $\left(N_{B} / 2\right)$ segments of type $B$, form a spherical droplet of radius $R_{2}$, and the central A-subchain, consisting of $N_{A}$ freely jointed segments of type A, assumes an arc-shape conformation whose ends are connected by a diameter $L_{2}=2 R_{2}$ of the droplet (see Figure 1b).

In both cases the effects of volume exclusion between segments were neglected: it should be particularly emphasized that the A-segments were allowed to enter the domain occupied by 


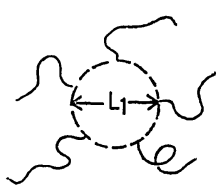

(a)

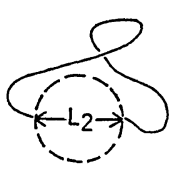

(b)

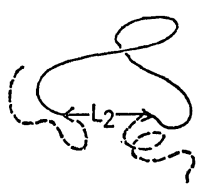

(c)
Figure 1. Statistical models-(a) for a micelle consisting of $f$ molecules of AB-diblock copolymer (star-shape model); and (b) for a BAB-triblock copolymer molecule, in which two B-subchains undergo intramolecular association, forming a droplet (arc-shape model), and for that (c) which has the usual conformation. The solvent is assumed to be good toward A-subchains (solid lines) but poor toward B-subchains (dashed lines).

the B-segments. Apparently this assumption is somehow contradictory to the hypothesis of the intrachain phase separation, and is admittedly too crude to describe the features of the conformational anomalies anticipated for block copolymer molecules. This is particularly important for the BAB-triblock copolymer system since, so far as the contribution from the Asubchain is concerned, the arc-shape model would be indistinguishable from a model in which the two B-subchains do not necessarily undergo the intramolecular association but the A-subchain has, by some reason, a restricted end-to-end distance (see Figure 1c). Thus when the $P(\theta)$ functions of the two models shown in Figures $1 \mathrm{~b}$ and $1 \mathrm{c}$ are compared, their only difference is trivial contributions from the lesseasily visible B-subchains.

General Properties of $\boldsymbol{P}(\theta)$ Functions of Copolymer Systems

For a copolymer system composed of two different types of segments having different scattering powers $g_{i}(i=\mathrm{A}$ or $\mathrm{B})$, the particlescattering function $P(\theta)$ should be given by

$$
\begin{gathered}
P(\theta)=\sum_{i, j} g_{i} g_{j} G_{i j}(w) / \sum_{i, j} g_{i} g_{j} \\
G_{i j}(w)=\left\langle\sin \left(w r_{i j}\right) /\left(w r_{i j}\right)\right\rangle \\
w=(4 \pi / \lambda) \sin (\theta / 2)
\end{gathered}
$$

Here $\theta$ is the angle between the direction of the scattered light and that of the incident light; $r_{i j}$ is the distance between segments $i$ and $j$; $\lambda$ is the wave length of the light in the given medium; the summation in eq 1 is to be taken over all the segment pairs; and $\langle\cdots\rangle$ denotes the average taken over all possible conformations of the system, whenever it has internal degrees of conformational freedom.

The segment $i$ (or $j$ ) may be either one of those belonging to $A$ or $B$ segments (therefore the factor $g_{i}$ should be proportional to $\nu_{\mathrm{A}} m_{\mathrm{A}}$ or $\nu_{B} m_{B}$, the choice depending on whether the segment $i$ belongs to the A-subchain or the B-subchain, respectively). Here $\nu_{A}$ (or $\nu_{B}$ ) and $m_{\mathrm{A}}$ (or $m_{\mathrm{B}}$ ) are the specific refractive increments of homopolymer A (or B) in the given medium and the molecular weight of the monomer $A$ (or B), respectively. The specific refractive increment $\nu$ of the copolymer system is approximated by a linear function of (average) composition: ${ }^{7,8}$

$$
\nu=x \nu_{\mathrm{A}}+(1-x) \nu_{\mathrm{B}}
$$

where $x$ is the (average) weight fraction of monomer $\mathbf{A}$ in the copolymer system. Then the terms in eq 1 may be regrouped into three parts:

$$
\begin{gathered}
P(\theta)=\alpha^{2} P_{\mathrm{A}}(\theta)+\beta^{2} P_{\mathrm{B}}(\theta)+2 \alpha \beta P_{\mathrm{AB}}(\theta) \\
\alpha=\nu_{\mathrm{A}} x / \nu ; \beta=\nu_{\mathrm{B}}(1-x) / \nu \\
P_{K}(\theta)=\left(f N_{K}\right)^{-2} \sum_{i, j}^{K} G_{i j}(w) ; K=\mathrm{A} \text { or B } \\
\boldsymbol{P}_{\mathrm{AB}}(\theta)=\left(f^{2} N_{\mathrm{A}} N_{\mathrm{B}}\right)^{-1} \sum_{i}^{\mathrm{A}} \sum_{j}^{\mathrm{B}} G_{i j}(w)
\end{gathered}
$$

where $f N_{K}$ is the total number of $K$-segments $(K=\mathrm{A}$ or $\mathrm{B})$ belonging to a micelle of $f$ molecules.

An apparent mean-square radius of gyration $\left\langle s^{2}\right\rangle_{\text {app }}$ of copolymer is defined from the initial slope of $P(\theta)$ versus $w^{2}$ plot by

$$
\begin{gathered}
\boldsymbol{P}(\theta)=1-\left(w^{2} / 3\right)\left\langle s^{2}\right\rangle_{\mathrm{app}}+\cdots \\
\left\langle\boldsymbol{s}^{2}\right\rangle_{\mathrm{app}}=\sum_{i, j} \boldsymbol{g}_{i} g_{j}\left\langle\boldsymbol{r}_{i j}{ }^{2}\right\rangle / \sum_{i, j} g_{i} \boldsymbol{g}_{j} .
\end{gathered}
$$

Again the quantity $\left\langle s^{2}\right\rangle_{\text {app }}$ may be regrouped into three parts ${ }^{9}$ as

$$
\begin{gathered}
\left\langle s^{2}\right\rangle_{\mathrm{app}}=\alpha^{2}\left\langle s^{2}\right\rangle_{\mathrm{A}}+\beta^{2}\left\langle s^{2}\right\rangle_{\mathrm{B}}+2 \alpha \beta\left\langle s^{2}\right\rangle_{\mathrm{AB}} \\
\left\langle s^{2}\right\rangle_{K}=(1 / 2)\left(f N_{K}\right)^{-2} \sum_{i, j}^{K}\left\langle r_{i j}{ }^{2}\right\rangle_{K} ; K=\mathrm{A} \text { or B } \\
\left\langle s^{2}\right\rangle_{\mathrm{AB}}=(1 / 2)\left(f^{2} N_{\mathrm{A}} N_{\mathrm{B}}\right)^{-1} \sum_{i}^{\mathrm{A}} \sum_{j}^{\mathrm{B}}\left\langle r_{i j}{ }^{2}\right\rangle_{\mathrm{AB}}
\end{gathered}
$$

The function $P(\theta)$ for a homopolymer chain can 
be expressed as a universal function of a variable $X=w^{2}\left\langle s^{2}\right\rangle$, where $\left\langle s^{2}\right\rangle$ is the mean square radius of gyration defined by eq 8 and 9 . By analogy with this variable, a corresponding variable $X$ for a copolymer chain was introduced which again can be regrouped into three terms:

$$
\begin{gathered}
X \equiv w^{2}\left\langle s^{2}\right\rangle_{\mathrm{app}}=\alpha^{2} X_{\mathrm{A}}+\beta^{2} X_{\mathrm{B}}+2 \alpha \beta X_{\mathrm{AB}} \\
X_{K}=w^{2}\left\langle s^{2}\right\rangle_{K} ; K=\mathrm{A} \text { or B } \\
X_{\mathrm{AB}}=w^{2}\left\langle s^{2}\right\rangle_{\mathrm{AB}} .
\end{gathered}
$$

In eq 5,10 and 12 , the terms with the subscripts $A$ and $B$ represent, respectively, the contributions from A- and B-subchains, and those with the double subscript $\mathrm{AB}$ represent the cross terms. The corresponding terms in the quantities $P(\theta),\left\langle s^{2}\right\rangle_{\text {app }}$ and $X$ may be correlated with one another by equations similar to eq 8 and 12 .

To calculate $P(\theta)$ for the present models, the following approximation $^{6,10-12}$ for $G_{i j}(w)$ was employed as necessary:

$$
G_{i j}(w) \cong \exp \left(-w^{2}\left\langle r_{i j}{ }^{2}\right\rangle / 6\right)
$$

The approximation may be fully justified for a random-flight chain, wherein the distribution function of a vector $\boldsymbol{r}_{i j}$ is Gaussian; and it is also justified for most flexible chains with certain limitations. ${ }^{6,10-12}$ The validity of applying eq 12 to the present models was examined by estimating the next higher order terms in eq 2 , which involve the fourth moment of the vector, $\left\langle r_{i j}{ }^{4}\right\rangle$. It was found that the approximation is good for the present models when the value of $X$ is small: the error is less than a few per cent, even when $X$ is as large as 5 .

Star-shape Model for a Micelle Consisting of $f$ AB-diblock Molecules

For this model (see Figure 1a) the contribution from A-subchains is calculated as follows. The second moment of a vector, $\boldsymbol{r}_{i j}$, connecting segments $i$ and $j$ both on A-subchain(s) is given by

$$
\begin{aligned}
\left\langle r_{i j}{ }^{2}\right\rangle_{\mathrm{A}}= & |i-j| b_{\mathrm{A}}{ }^{2} ; i \text { and } j \text { on the same branch } \\
\left\langle r_{i j}{ }^{2}\right\rangle= & (i+j) b_{\mathrm{A}}{ }^{2}+2 R_{1}{ }^{2} \text {; } \\
& \quad i \text { and } j \text { on the different branches }
\end{aligned}
$$

where $b_{\mathrm{A}}{ }^{2}$ is the mean-square length of an Asegment vector. Then by definition, eq 11a, the term $\left\langle s^{2}\right\rangle_{\mathrm{A}}$ is given by

$$
\left\langle s^{2}\right\rangle_{\mathrm{A}}=\frac{N_{\mathrm{A}} b_{\mathrm{A}}{ }^{2}}{6}\left[\frac{3 f-2}{f}\right]+R_{1}{ }^{2}\left[\frac{f-1}{f}\right]
$$

Finally from eq $7 \mathrm{a}, 14,15 \mathrm{a}$, and $15 \mathrm{~b}, \boldsymbol{P}_{\mathrm{A}}(\theta)$ can be expressed as:

$$
\begin{gathered}
P_{\mathrm{A}}(\theta)=\frac{2}{f Y_{\mathrm{A}}{ }^{2}}\left[\exp \left(-Y_{\mathrm{A}}\right)+Y_{\mathrm{A}}-1\right. \\
\left.+\frac{f-1}{2} \exp \left(-u_{\mathrm{B}}{ }^{2} / 3\right)\left\{\exp \left(-Y_{\mathrm{A}}\right)-1\right\}^{2}\right] \\
Y_{\mathrm{A}}=w^{2}\left(N_{\mathrm{A}} b_{\mathrm{A}}{ }^{2} / 6\right) \\
u_{\mathrm{B}}{ }^{2}=w^{2} R_{1}{ }^{2}
\end{gathered}
$$

When $R_{1}=0$, eq 17 reduces to the $P(\theta)$ function of a regular star model with $f$ branches given earlier by Benoit. ${ }^{14}$

For the contribution from the spherical core composed of B-subchains, the sphere is assumed to have a uniform density. Hence;

$$
\left\langle s^{2}\right\rangle_{\mathrm{B}}=(3 / 5) R_{1}{ }^{2}
$$

For the $P_{\mathrm{B}}(\theta)$, the $P(\theta)$ function for a sphere ${ }^{15}$ can be directly applied:

$$
P_{\mathrm{B}}(\theta)=\left[\left(3 / u_{\mathrm{B}}^{3}\right)\left(\sin u_{\mathrm{B}}-u_{\mathrm{B}} \cos u_{\mathrm{B}}\right)\right]^{2}
$$

For the calculation of the AB-cross terms, one obtains

$$
\left\langle r_{i j}{ }^{2}\right\rangle_{\mathrm{AB}}=i b_{\mathrm{A}}{ }^{2}+R_{1}{ }^{2}+r_{j}{ }^{2}
$$

where $r_{j}$ is the distance between a position $j$ and the center of the spherical core composed of the B-subchains. Here it has been assumed that the segment $i$ is found on an A-subchain and $j$ in the core. From eq $11 \mathrm{c}$ and 21 , the term $\left\langle s^{2}\right\rangle_{\mathrm{AB}}$ becomes

$$
\left\langle s^{2}\right\rangle_{\mathrm{AB}}=(4 / 5) R_{1}{ }^{2}+N_{\mathrm{A}} b_{\mathrm{A}}{ }^{2} / 4
$$

Then for the calculation of the $\boldsymbol{P}_{\mathrm{AB}}(\theta)$, eq 14 and 21 are employed to obtain

$$
\begin{aligned}
P_{\mathrm{AB}}(\theta)= & \left(3 / Y_{\mathrm{A}}\right)\left[1-\exp \left(-Y_{\mathrm{A}}\right)\right] \exp \left(-{u_{\mathrm{B}}}^{2} / 6\right) \\
& \times\left(\sqrt{6} / u_{\mathrm{B}}\right)^{3} I_{1}\left(u_{\mathrm{B}} / \sqrt{6}\right) \\
& I_{1}(y)=\int_{0}^{y} t^{2} \exp \left(-t^{2}\right) \mathrm{d} t
\end{aligned}
$$

The particle scattering function $P(\theta)$ of the whole micelle is constructed by combining eq 5 with eq 17,20 , and 23 . 
On the other hand, from eq $13,16,19$, and 22 ,

$$
\begin{gathered}
X_{\mathrm{A}}=\frac{3 f-2}{Y_{\mathrm{A}}+\frac{f-1}{f} u_{\mathrm{B}}{ }^{2}} \\
X_{\mathrm{B}}=(3 / 5) u_{\mathrm{B}}{ }^{2} \\
X_{\mathrm{AB}}=(3 / 2) Y_{\mathrm{A}}+(4 / 5) u_{\mathrm{B}}{ }^{2}
\end{gathered}
$$

The quantity $Y_{\mathrm{A}}$ may be independently determined by measuring the radius of gration, $N_{\mathrm{A}} b_{\mathrm{A}}{ }^{2} / 6$, of the corresponding precursor homopolymer $\mathrm{A}$ in the given medium; whilst the quantity $u_{\mathrm{B}}{ }^{2}$ may be correlated with $Y_{\mathrm{A}}$ by using a ratio

$$
\gamma^{2}=R_{1}^{2} / N_{\mathrm{A}}{b_{\mathrm{A}}}^{2}=u_{\mathrm{B}}{ }^{2} / 6 Y_{\mathrm{A}}
$$

as an adjustable parameter.

\section{Arc-Shape Model for a BAB-triblock Copolymer Molecule}

For this model (see Figure 1b), it is assumed that the end-to-end vector $L_{2}$ of A-subchain is fixed on a diameter $\boldsymbol{L}=2 \boldsymbol{R}_{2}$ of the spherical droplet composed of two B-subchains. In general the distribution function $W\left(\boldsymbol{r}_{i j} \mid \boldsymbol{L}\right)$ of a vector $\boldsymbol{r}_{i j}$ on a chain having $N$ freely jointed segments and a restricted end-to-end vector $L$ may be given by

$$
W\left(\boldsymbol{r}_{i j} \mid \boldsymbol{L}\right)=W\left(\boldsymbol{r}_{i j}, \boldsymbol{L}\right) / W(\boldsymbol{L})
$$

where $W\left(\boldsymbol{r}_{i j}, \boldsymbol{L}\right)$ is the bivariate probability density for an unrestricted chain and $W(\boldsymbol{L})$ is its probability density for an unrestricted chain and $W(\boldsymbol{L})$ is its probability density of the endto-end vector $L^{16} \quad$ They are respectively given as follows:

$$
\begin{aligned}
W\left(\boldsymbol{r}_{i j}, \boldsymbol{L}\right) & =\left[\frac{3}{2 \pi b^{2}}\right]^{3}\{k(N-k)\} \\
\times \exp & \left\{-\frac{3\left(N r_{i j}{ }^{2}+k L^{2}-2 k \boldsymbol{r}_{i j} \boldsymbol{L}\right)}{2 b^{2} k(N-k)}\right\} \\
W(\boldsymbol{L}) & =\left[\frac{3}{2 \pi N b^{2}}\right]^{3 / 2} \exp \left\{-\frac{3 L^{2}}{2 N b^{2}}\right\}
\end{aligned}
$$

where $k=|i-j|, b^{2}$ is the mean-square length of a segment vector, and $N$ is the number of such segments in the chain.

The distribution function $W\left(\boldsymbol{r}_{i j} \mid \boldsymbol{L}\right)$ can be directly applied to the calculation of the term $\left\langle r_{i j}{ }^{2}\right\rangle_{\mathrm{A}}$ of this arc-shape model:

$$
\begin{aligned}
\left\langle\boldsymbol{r}_{i j}{ }^{2}\right\rangle_{\mathrm{A}} & =\int \boldsymbol{r}_{i j}{ }^{2} W\left(\boldsymbol{r}_{i j} \mid \boldsymbol{L}\right) \mathrm{d} \boldsymbol{r}_{i j} \\
& =|i-j| b_{\mathrm{A}}{ }^{2}\left[1+\left(|i-j| / N_{\mathrm{A}}\right)\left(l^{2}-1\right)\right]
\end{aligned}
$$

$$
l^{2}=L_{2}^{2} / N_{\mathrm{A}} b_{\mathrm{A}}{ }^{2}
$$

From eq 11a and 27, the term $\left\langle s^{2}\right\rangle_{\mathrm{A}}$ becomes

$$
\left\langle s^{2}\right\rangle_{\mathrm{A}}=\frac{1}{2}\left(1+l^{2}\right) \frac{N_{\mathrm{A}}{b_{\mathrm{A}}}^{2}}{6}
$$

Here the parameter $l^{2}$ is a measure for the restriction on the end-to-end distance of the Asubchain. Then from eq $7 \mathrm{a}, 14$, and 27 , one has the following three forms for the $P_{\mathrm{A}}(\theta)$, which depend on the value of $l^{2}$ :

$$
\begin{aligned}
P_{\mathrm{A}}(\theta)= & 2\left(2 \varepsilon Y_{\mathrm{A}}\right)^{-1 / 2}[1-(1 / 4 \varepsilon)] \mathrm{e}^{-Y_{\mathrm{A}} / 8 \varepsilon} \\
& \times\left\{I _ { 2 } \left(\sqrt{2 \varepsilon Y_{\mathrm{A}}}-\sqrt{\left.Y_{\mathrm{A}} / 8 \varepsilon\right)}+I_{2}\left(\sqrt{\left.Y_{\mathrm{A}} / 8 \varepsilon\right)}\right\}\right.\right. \\
& -\left(2 \varepsilon Y_{\mathrm{A}}\right)^{-1}\left[\mathrm{e}^{(2 \varepsilon-1) Y_{\mathrm{A}}}-1\right] ;\left(0<l^{2}<1\right) \quad(30 \mathrm{a}) \\
P_{\mathrm{A}}(\theta)= & 2 Y_{\mathrm{A}}^{-2}\left[\mathrm{e}^{-Y_{\mathrm{A}}}-1+Y_{\mathrm{A}}\right] ;\left(l^{2}=1\right) \\
P_{\mathrm{A}}(\theta)= & 2\left(2|\varepsilon| Y_{\mathrm{A}}\right)^{-1 / 2}[1-(1 / 4 \varepsilon)] \mathrm{e}^{-Y_{\mathrm{A}} / 8 \varepsilon} \\
& \times\left\{I_{3}\left(\sqrt{2|\varepsilon| Y_{\mathrm{A}}}+\sqrt{Y_{\mathrm{A}} / 8|\varepsilon|}\right)-I_{3}\left(\sqrt{Y_{\mathrm{A}} / 8|\varepsilon|}\right)\right. \\
& -\left(2 \varepsilon Y_{\mathrm{A}}\right)^{-1}\left[\mathrm{e}^{(2 \varepsilon-1) Y_{\mathrm{A}}}-1\right] ;\left(l^{2}>1\right)
\end{aligned}
$$

where the quantities $Y_{\mathrm{A}}, \varepsilon, I_{2}(y)$, and $I_{3}(y)$ are respectively given as follows

$$
\begin{aligned}
Y_{\mathrm{A}} & =w^{2}\left(N_{\mathrm{A}} b_{\mathrm{A}}{ }^{2} / 6\right) \\
\varepsilon & =\left(1-l^{2}\right) / 2 \\
I_{2}(y) & =\int_{0}^{y} \exp \left(t^{2}\right) \mathrm{d} t \\
I_{3}(y) & =\int_{0}^{y} \exp \left(-t^{2}\right) \mathrm{d} t
\end{aligned}
$$

Apparently eq $30 \mathrm{~b}$ is the well-known Debye equation $^{6}$ for a Gaussian chain, since $Y_{\mathrm{A}}=X_{\mathrm{A}}$. When $l^{2}=0$, eq 30 a reduces to the $P(\theta)$ function of a circular chain, which was given earlier by Casassa. ${ }^{17}$

$$
\begin{gathered}
P_{\mathrm{A}}(\theta)=\left(2 / X_{\mathrm{A}}\right)^{1 / 2} \mathrm{e}^{-X_{\mathrm{A}} / 2}\left\{I_{2}\left(\sqrt{X_{\mathrm{A}} / 2}\right)\right\} \\
X_{\mathrm{A}}=Y_{\mathrm{A}} / 2
\end{gathered}
$$

The $P_{\mathrm{A}}(\theta)$ function for the case where $l^{2}>1$ was also derived by various other authors, ${ }^{18-20}$ their models being somewhat different, but their results were essentially the same as for eq 30c.

For the contribution from the droplet composed of B-subchains, eq 19 and 20 apply to this arc-shape model as well (with a minor revision of replacing $R_{1}$ by $R_{2}$ ). It should be noted that the intrachain association of the two B-subchains automatically results in the restriction on the end-to-end distance of the A-subchain: therefore 
Light Scattering of Block Copolymer Solutions

$$
\begin{gathered}
u_{\mathrm{B}}{ }^{2}=(3 / 2) l^{2} Y_{\mathrm{A}} \\
\left\langle s^{2}\right\rangle_{\mathrm{B}}=(9 / 5)\left[l^{2} /\left(1+l^{2}\right)\right]\left\langle s^{2}\right\rangle_{\mathrm{A}}
\end{gathered}
$$

Finally for the contribution of the AB-cross terms, one obtains

$$
\left\langle r_{i j}{ }^{2}\right\rangle_{\mathrm{AB}}=i b_{\mathrm{A}}{ }^{2}\left(1-l^{2}\right)\left(1-i / N_{\mathrm{A}}\right)+{R_{2}}^{2}+r_{j}{ }^{2}
$$

where $r_{j}$ is the distance between an arbitrary position $j$ in the droplet and its center. Then the term $\left\langle s^{2}\right\rangle_{A B}$ becomes

$$
\left\langle s^{2}\right\rangle_{\mathrm{AB}}=\frac{1}{2}\left(1-l^{2}\right) \frac{N_{\mathrm{A}} b_{\mathrm{A}}{ }^{2}}{6}+\frac{4}{5} R_{2}{ }^{2}
$$

From eq 7c, 14, and 33, the following three forms for the $\boldsymbol{P}_{\mathrm{AB}}(\theta)$ can be obtained, each again depending on the value of $l^{2}$ :

$$
\begin{gathered}
P_{\mathrm{AB}}(\theta)=3\left\{4 /(1-2 \varepsilon) Y_{\mathrm{A}}\right\}^{3 / 2}\left\{I_{1}\left(\sqrt{(1-2 \varepsilon) Y_{\mathrm{A}} / 4}\right\}\right. \\
\times\left(2 / \varepsilon Y_{\mathrm{A}}\right)^{1 / 2}\left\{I _ { 2 } \left(\sqrt{\left.\left.\varepsilon Y_{\mathrm{A}} / 2\right)\right\}} \mathrm{e}^{-Y_{\mathrm{A}} / 4} ;\right.\right. \\
\left(0<l^{2}<1\right) \\
P_{\mathrm{AB}}(\theta)=3\left(4 / Y_{\mathrm{A}}\right)^{3 / 2}\left\{I_{1}\left(\sqrt{Y_{\mathrm{A}} / 4}\right)\right\} \mathrm{e}^{-Y_{\mathrm{A}} / 4} ; \\
\left(l^{2}=1\right) \\
P_{\mathrm{AB}}(\theta)=3\left\{4 /(1-2 \varepsilon) Y_{\mathrm{A}}\right\}^{3 / 2}\left\{I_{1}\left(\sqrt{(1-2 \varepsilon) Y_{\mathrm{A}} / 4}\right)\right. \\
\times\left(2 /|\varepsilon| Y_{\mathrm{A}}\right)^{1 / 2}\left\{I_{3}\left(\sqrt{\left.|\varepsilon| Y_{\mathrm{A}} / 2\right)}\right\} \mathrm{e}^{-Y_{\mathrm{A}} / 4} ;\right. \\
\left(l^{2}>1\right)
\end{gathered}
$$

Thus combining eq 5 with eq 30, 20, and 35, the function $P(\theta)$ for an arc-shape model is obtained. Also from eq 13, 29, 19, and 34, one has

$$
\begin{aligned}
& X_{\mathrm{A}}=\frac{1}{2}\left(1+l^{2}\right) Y_{\mathrm{A}} \\
& X_{\mathrm{B}}=(3 / 5) u_{\mathrm{B}}{ }^{2} \\
& X_{\mathrm{AB}}=\frac{1}{2}\left(1-l^{2}\right) Y_{\mathrm{A}}+\frac{4}{5}{u_{\mathrm{B}}}^{2}
\end{aligned}
$$

The function $\boldsymbol{P}(\theta)$ can be given as a function of $X$, by using $l^{2}$ as an adjustable parameter.

\section{Behavior of the Models}

Here one is interested primarily in the cases wherein B-subchains are nearly invisible and the essential part of the Rayleigh scattering comes mainly from A-subchain(s), i.e., $\nu_{A} \gg \nu_{B}$ and hence $\beta / \alpha \cong 0$. Therefore one should examine the $P_{\mathrm{A}}(\theta)$ versus $X_{\mathrm{A}}$ relation for each model. Figure 2 illustrates the features of the reciprocal $P_{\mathrm{A}}(\theta)$ versus $\boldsymbol{X}_{\mathrm{A}}$ plots of the star-shape model with a few different values of $f$ and $\gamma^{2}$.

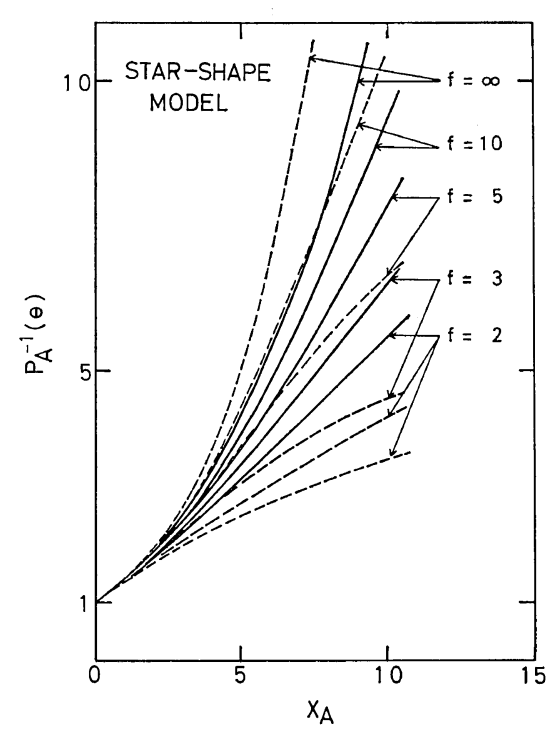

Figure 2. Reciprocal $P_{A}(\theta)$ versus $X_{A}$ plots calculated for the contribution from A-subchains of star-shape models with various values of $f$ as indicated: solid curves are those with $\gamma^{2}=0$ and dashed curves with $\gamma^{2}=0.5$; and three curves with $f=2$ represent those with $\gamma^{2}=0,0.5$ and 1.0 , respectively, from top to bottom. For details, see text.

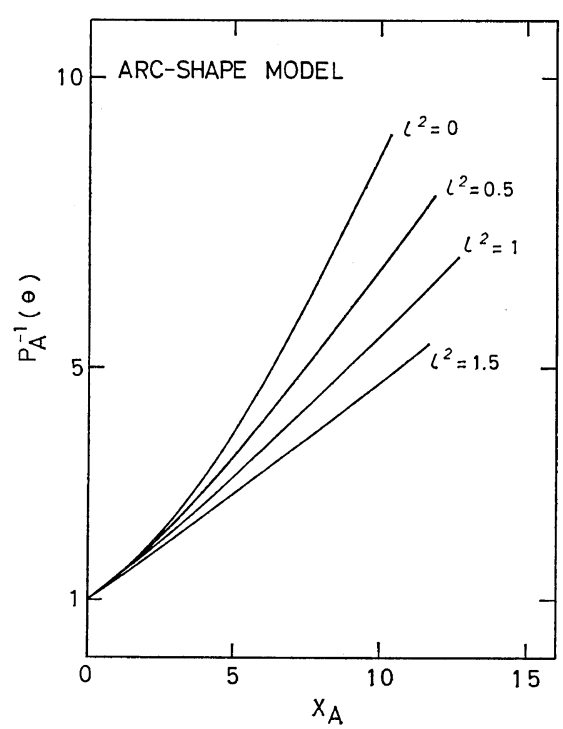

Figure 3. Reciprocal $P_{A}(\theta)$ versus $X_{A}$ plots calculated for the contribution from A-subchain of arc-shape model with various values of $l^{2}$ as indicated. For details, see text. 
Obviously, when the diameter of the core diminishes to zero $\left(\gamma^{2}=0\right)$, the $P_{\mathrm{A}}(\theta)$ reduces to that of a regular star model with $f$ branches. ${ }^{14}$ When $\gamma^{2}=0$ and $f=2$, the $P_{\mathrm{A}}(\theta)$ reduces to the Debye function $^{6}$ of a linear Gaussian chain. As $f$ increases, the reciprocal $\boldsymbol{P}_{\mathrm{A}}(\theta)$ becomes a more rapidly increasing function of $X_{\mathrm{A}}$, and the plot becomes more upwardly concave. Interestingly, the effect of the (invisible) core size reverses, as $f$ increases from five to ten: namely as the parameter $\gamma^{2}$ increases, the reciprocal $P_{\mathrm{A}}(\theta)$ becomes a less rapidly increasing function of $X_{\mathrm{A}}$ for the model with $f=5$; whilst it becomes a more rapidly increasing function of $X_{\mathrm{A}}$ for the model with $f=10$.

Figure 3 illustrates the features of the reciprocal $\boldsymbol{P}_{\mathrm{A}}(\theta)$ versus $\boldsymbol{X}_{\mathrm{A}}$ relationship of the arcshape model with a few differing values of $l^{2}$. When $l^{2}=1$, the plot corresponds approximately to that of an unrestricted Gaussian chain; ${ }^{6}$ when $l^{2}=0$, the plot becomes a more rapidly increasing function of $X_{\mathrm{A}}$, and coincides with that of a circular Gaussian chain. ${ }^{17}$ On the contrary, as $l^{2}$ increases, the plot shifts to the opposite direction: namely it becomes a more slowly increasing function of $X_{\mathrm{A}}$ and its curvature becomes more and more downwardly concave than that of a linear Gaussian chain. ${ }^{18}$

\section{EXPERIMENTAL PROCEDURE AND RESULTS}

\section{Materials and Methods}

Two samples of PST (A) and PMMA (B) block copolymers, one each of AB- and BABtype, and their precursor homo-PST samples were used. These samples were prepared by an anionic polymerization technique, which was described elsewhere in greater detail. ${ }^{2}$

Light scattering measurements in $p$-xylene solutions at $30^{\circ} \mathrm{C}$ were made by a Shimadzu light-scattering photometer of modified Brice type. The details were reported elsewhere. ${ }^{21}$ The solvent, $p \mathrm{XY}$, was carefully distilled twice immediately before use. Sample solutions were cleaned by centrifugation at $12000 \mathrm{rpm}$ for two hours before each measurement. A cylindrical cell having two optically flat surfaces in the path of the incident beam was employed. The angular variation of the scattered intensity or the Rayleigh ratio was determined at eleven different angles ranging from $30^{\circ}$ to $150^{\circ}$ with vertically polarized light of $436 \mathrm{~m} \mu$ wavelength. The apparatus constant was evaluated with pure benzene as a standard liquid, by taking $U_{\mathrm{u}}=$ $46.4 \times 10^{-6}$ for Rayleigh ratio at $90^{\circ}$ and $\rho_{\mathrm{u}}=$ 0.42 for the corresponding depolarization ${ }^{22}$ for the unpolarized light of $436 \mathrm{~m} \mu$ wavelength measured at $25^{\circ} \mathrm{C}$.

\section{Analysis of Light-Scattering Data}

Rayleigh ratios $R(\theta, c)$ at scattering angle $\theta$ from a solution with polymer concentration $c$ were analyzed according to Zimm's procedure ${ }^{23}$ in order to determine apparent molecular weight, $M_{\text {app }}$, apparent second virial coefficient, $\left(A_{2}{ }^{\prime}\right)_{\text {app }}{ }^{2}$, and $\left\langle s^{2}\right\rangle_{\text {app }}$ :

$$
\begin{aligned}
& \lim _{\theta \rightarrow 0} K c / R(\theta, c)=\left(M_{\mathrm{app}}\right)^{-1}+2\left(A_{2}{ }^{\prime}\right)_{\mathrm{app}} c+\cdots(37 \mathrm{a}) \\
& \lim _{c \rightarrow 0} K c / R(\theta, c)=\left(M_{\mathrm{app}}\right)^{-1}\left[1+\left(w^{2} / 3\right)\left\langle s^{2}\right\rangle_{\mathrm{app}}+\cdots\right]
\end{aligned}
$$

where $K$ is the optical constant. ${ }^{23}$ For homopolymer solutions $M_{\mathrm{app}},\left(A_{2}{ }^{\prime}\right)_{\mathrm{app}}$, and $\left\langle s^{2}\right\rangle_{\mathrm{app}}$ are the weight-average molecular weight $M_{w}$, the (light-scattering) second virial coefficient $A_{2}{ }^{\prime}$, and the ( $z$-average) mean-square radius of gration, respectively. ${ }^{2,7-9}$ Figures 4, 5, and 6 show the

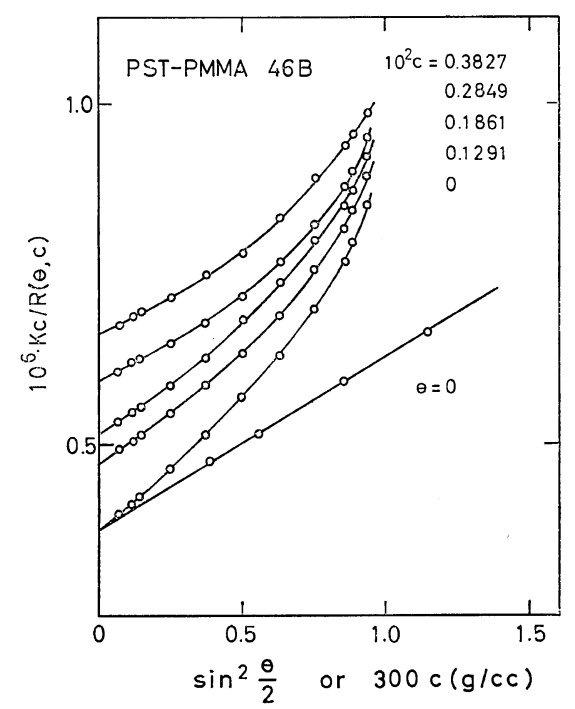

Figure 4. Plots of $K c / R(\theta, c)$ versus $\sin ^{2}(\theta / 2)$ measured at $30^{\circ} \mathrm{C}$ for $p \mathrm{XY}$ solutions of PST-PMMA diblock sample 46B with various concentrations as indicated. 


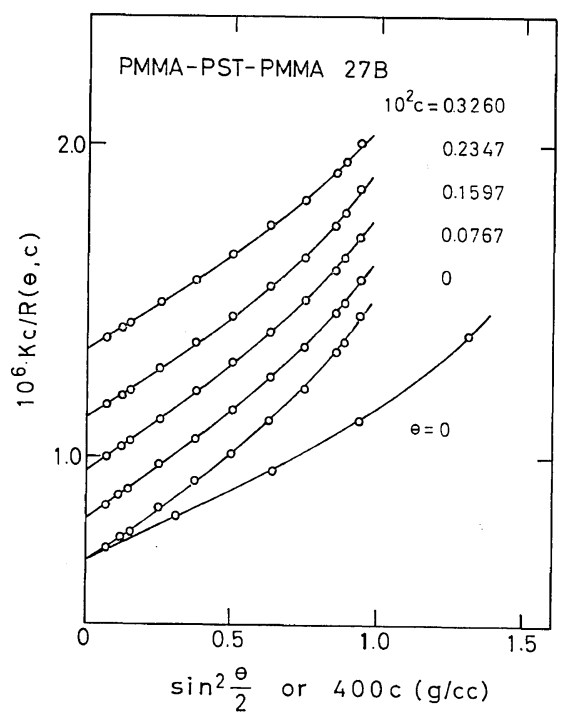

Figure 5. Plots of $K c / R(\theta, c)$ versus $\sin ^{2}(\theta / 2)$ measured at $30^{\circ} \mathrm{C}$ for $p \mathrm{XY}$ solutions of PMMA-PST -PMMA triblock sample 27B with various concentrations as indicated.

plots of $K c / R(\theta, c) v s \cdot \sin ^{2}(\theta / 2)$ for $p \mathrm{XY}$ solutions of the PST-PMMA diblock sample 46B, the PMMA-PST-PMMA triblock sample 27B and the homopolymer PST-27H (which is the precursor of the sample 27B), respectively, all measured at $30^{\circ} \mathrm{C}$. Table I summarizes the results obtained together with some other pertinent data. The $M_{\mathrm{app}}$ data suggest that the diblock sample 46B forms stable micelles, each consisting of about 7 to 10 molecules, whereas the triblock sample 27B appears to remain in the state of monomolecular dispersion.

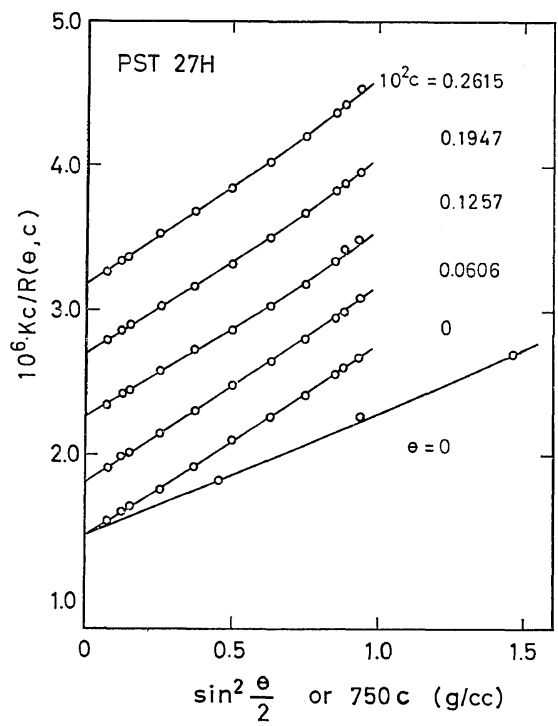

Figure 6. Plots of $K c / R(\theta, c)$ versus $\sin ^{2}(\theta / 2)$ measured at $30^{\circ} \mathrm{C}$ for $p \mathrm{XY}$ solutions of precursor PST $27 \mathrm{H}$ with various concentrations as indicated.

\section{Angular Dependence of Rayleigh Scattering}

The plots of $K c / R(\theta, c)$ versus $\sin ^{2}(\theta / 2)$, extrapolated to zero polymer concentration, reflect the behavior of the reciprocal $\boldsymbol{P}(\theta)$ functions of these systems. The plot for the diblock sample 46B, shown in Figure 4, exhibits an anomalous upward curvature. Figure 5 shows a similar plot for the triblock sample 27B: an anomalous curvature is also seen, although the extent is less marked. These anomalous curvatures are to be contrasted with the plot of the homo-PST 27H in Figure 6, which shows no such anomalies. As mentioned, the major contribution to the $\boldsymbol{P}(\theta)$ functions of the present systems comes

Table I. Summary of light-scattering data on two PST and PMMA block copolymer samples and their precursor homo-PST samples in $p$-xylene solutions at $30^{\circ} \mathrm{C}$

\begin{tabular}{|c|c|c|c|c|c|c|c|c|}
\hline Somple code & $\begin{array}{l}\text { ST-weight } \\
\text { fraction }\end{array}$ & $10^{-4} M_{w}{ }^{2}$ & $\nu$ & $\alpha$ & $\beta$ & $10^{-4} M_{\mathrm{app}}$ & $\begin{array}{c}10^{12} \\
\left\langle s^{2}\right\rangle_{\text {app }}\end{array}$ & $\begin{array}{c}10^{4} \\
\left.\left(A_{2}\right)^{\prime}\right)_{\text {app }}\end{array}$ \\
\hline PST $46 \mathrm{H}$ & 1.00 & 14.0 & 0.114 & - & - & 14.7 & 2.4 & 4.67 \\
\hline PST-PMMA 46B & 0.45 & 30.0 & $0.055_{3}$ & 0.926 & 0.074 & $266.5^{\mathrm{b}}$ & 14.0 & 0.383 \\
\hline PST $27 \mathrm{H}$ & 1.00 & 67.1 & 0.114 & - & - & 69.0 & 14.3 & 3.15 \\
\hline PMMA-PST-PMMA 27B & B $\quad 0.41$ & 147.4 & $0.051_{1}$ & 0.915 & 0.085 & 149.5 & 14.8 & 0.898 \\
\hline
\end{tabular}

a For homo-PST samples, the values were determined in 2-butanone at $30^{\circ} \mathrm{C}$. For block copolymer samples the values were determined from $M_{\text {app }}$ data obtained in several different solvents. ${ }^{7,8,2}$

b It was found that $10^{-4} M_{\mathrm{app}}=32.6$ at $30^{\circ} \mathrm{C}$ in $\mathrm{TOL}$, in which the block copolymers do not form micelles. 
from PST (=A)-subchains. Hence these curves may be compared with the $P_{\mathrm{A}}(\theta)$ versus $X_{\mathrm{A}}$ relations such as are shown in Figures 2 and 3. The data including those of the homo-PST $27 \mathrm{H}$ are at least in qualitative agreement with the behavior of the theoretical $\boldsymbol{P}_{\mathrm{A}}(\theta)$ functions derived from the models presented above.

Judging from the $M_{\text {app }}$ data and the behavior of the $P(\theta)$ function, one can be almost certain that the diblock sample 46B forms stable micelles in $p \mathrm{XY}$ solution. Therefore it would be interesting to compare the $\boldsymbol{P}(\theta)$ data with those of regular star-shape PST's. Likewise it would be interesting to compare the $P(\theta)$ data of the triblock sample 27B with those of circular-shape PST's. Unfortunately however, no such data are available at the moment, as far as the authors are aware. The latter samples (circularshape polymers) in particular have not been successfully synthesized so far, although an attempt now underway stands a very good chance of success. ${ }^{24}$ On the other hand, a few articles have been published on the dilute solution properties of star-shape PST's. ${ }^{25-27}$ However, again as far as the authors are aware, no $P(\theta)$ data have been published so far.

In this connection, it is interesting to note a recent report of Nagasawa, et al. ${ }^{28,29}$ on combshape PST's. One of the samples, J-2', had a short backbone and relatively long branches and might therefore be regarded as practically starshaped. Therefore its behavior should resemble, to some extent, that of a star-shape PST. Its reciprocal $P(\theta)$ function would be expected to show an anomalous upward curvature as predicted for a star-shape model ( $c f$., Figure 2). However the plot of $K c / R(\theta, c=0)$ versus $\sin ^{2}(\theta / 2)$ of the comb-shape PST sample $\mathrm{J}-2^{\prime}$ measured in cyclohexane at $33^{\circ} \mathrm{C}$, i.e., the $\theta$ condition, is a straight line over all the range of $\theta$ observed, and it does not show anomalous curvature ( $c f$., Figure 2 in ref 27). It should be noted, however, that the slope of the plot is considerably larger than that expected from a theoretical $P(\theta)$ function for the comb-shape model (eq 8 in ref 27). This latter has been calculated on the basis of the assumption essentially the same as that employed here, i.e., by assuming the same statistical segment as for linear unrestricted polymer chain. This discrepancy between the theory and experiment might be due to an inhomogeneity of the branch numbers of the individual comb-shape molecules: the inhomogeneity might obscure the curvature of the reciprocal $P(\theta)$ function but make its apparent initial slope larger than the value it should otherwise have. The situation resembles to some extent the $P(\theta)$ function of a polydisperse linear polymer chain, which deviates considerably from the Debye function and gives the $z$-average $\left\langle s^{2}\right\rangle_{\text {app }}$ value from its initial slope. It should be mentioned however that Nagasawa, et al., gave a different interpretation of this discrepancy. ${ }^{28}$ More accurate $\boldsymbol{P}(\theta)$ data on welldefined star-shape or comb-shape polymers will be necessary before a definite conclusion on this point can be reached.

\section{DISCUSSION}

From the data shown in Figures 4 and 5, the reciprocal $P(\theta)$ versus $X$ relations are readily obtained from eq 37 and 12 . The results are shown in Figures 7 and 8 . Although the major contribution to the $P(\theta)$ function comes from the

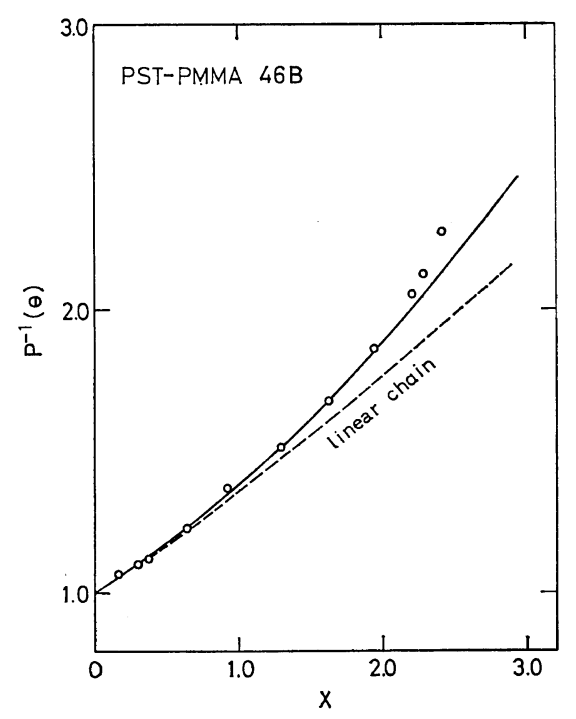

Figure 7. Reciprocal $P(\theta)$ versus $X$ plots for PSTPMMA diblock sample $46 \mathrm{~B}$ in $p \mathrm{XY}$ at $30^{\circ} \mathrm{C}$; circles represent experimental data; solid curve is calculated for a star-shape model with $f=8$ and $\gamma^{2}=0.63$; and dashed curve is for a linear Gaussian chain. For details, see text. 
A-subchains, the contributions from other terms, especially from the AB-cross terms, are not entirely negligible, as judged from the values of $\alpha$ and $\beta$ for the present systems. Therefore a closer examination of the theory and experiments demands an analysis of these minor terms also: they often amount to about $10-\%$ of the overall $\boldsymbol{P}(\theta)$ function.

First the PST-PMMA diblock copolymer system is examined. From the little evidence given so far, ${ }^{5}$ it is evident that in $p \mathrm{XY}$ at $30^{\circ} \mathrm{C}$ the sample $46 \mathrm{~B}$ undergoes intermolecular association and forms stable micelles even at infinite dilution. Particularly from the $M_{\text {app }}$ data of this sample obtained in $p X Y$ and in toluene (TOL), it is reasonable to assign the value of $f=8(c f$., Table I). On the other hand, from the $\left\langle s^{2}\right\rangle_{\text {app }}$ data of the sample $46 \mathrm{~B}$ and its precursor PST $46 \mathrm{H}$, one obtains $X=14.0 \times 10^{-12} w^{2}$ and $Y_{\mathrm{A}}=2.4 \times 10^{12} w^{2}$, respectively; and then, by eq 12 and $25, r^{2}=R_{1}{ }^{2} / N_{\mathrm{A}} b_{\mathrm{A}}{ }^{2}=0.63$. This value of $\gamma^{2}$ appears to be a little too large but not unreasonable, since the sample $46 \mathrm{~B}$ has a nearly equimolar composition, i.e., its PST- and PMMA-subchains have nearly the same molecular weight; and $p \mathrm{XY}$ is a poor solvent toward PMMA but a good solvent toward PST. Using these values and other pertinent data ( $c f$., Table I), the $P(\theta)$ versus $X$ relationship can be calculated from eq 5, 17, 20, and 23 for the star-shape model with the given parameters. Figure 7 shows the results in the form of the reciprocal $\boldsymbol{P}(\theta)$ versus $\boldsymbol{X}$ plot. The plot is compared with the experimental data for the sample $46 \mathrm{~B}$ in $p \mathrm{XY}$ at $30^{\circ} \mathrm{C}$. The agreement is good except in the region of large scattering angles. Judging from all these data of $M_{\mathrm{app}},\left\langle\mathrm{s}^{2}\right\rangle_{\mathrm{app}}$ and the $\boldsymbol{P}(\theta)$ versus $\boldsymbol{X}$ relation, it may be concluded that the star-shape model provides a good description of PST-PMMA diblock copolymers in $p X Y$ solutions. A definite number of PST-PMMA diblock molecules form oil-inoil type micelles, in which PMMA subchains constitute the core and soluble PST-subchains wrap around the core and stabilize the micelle. The size and the stability of such micelles are apparently dependent on the molecular weight and the composition of the block copolymers or, in other words, on the molecular weights of the individual constituent subchains.

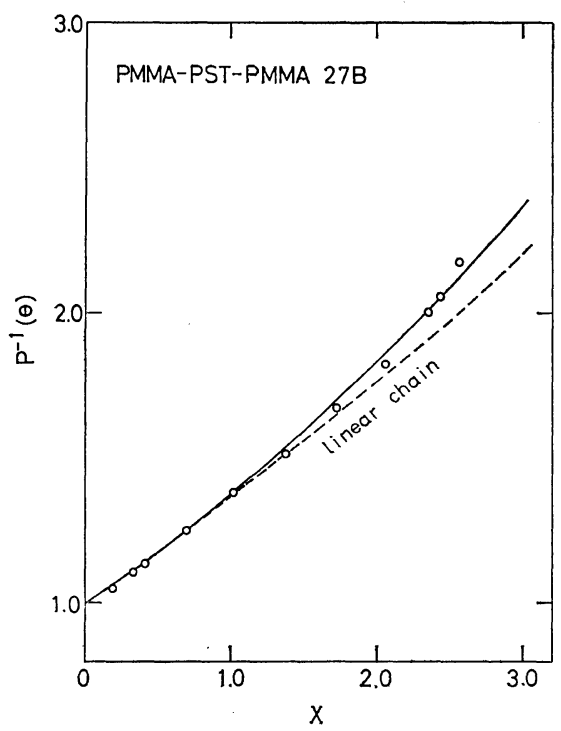

Figure 8. Reciprocal $P(\theta)$ versus $X$ plots for PMMA -PST-PMMA triblock sample 27B in $p X Y$ at $30^{\circ} \mathrm{C}$ : Circles represent experimental data; solid curve is calculated for an arc-shape model with $l^{2}=0.5$; and dashed cvrve is for a linear Gaussian chain. For details, see text.

On the contrary, the results of the PMMAPST_PMMA triblock copolymer system appear to be somewhat contradictory. First of all, the $M_{\text {app }}$ data suggest that the triblock sample 27B does not undergo the intermolecular association, in spite of the fact that its PMMAsubchains have sufficiently large molecular weight: the molecular weight of a PMMA-subchain of the triblock sample $27 \mathrm{~B}$ is about $43 \times$ $10^{4}$, whilst that of the micelle-forming diblock sample $46 \mathrm{~B}$ is abont $15 \times 10^{4}$ and that of another diblock sample 47B, which was found to form a micelle consisting of about 6 molecules, ${ }^{5}$ is only about $5 \times 10^{4}$. From the $\left\langle s^{2}\right\rangle_{\text {app }}$ data of the sample $27 \mathrm{~B}$ and its precursor PST $27 \mathrm{H}$, one has $X=14.8 \times 10^{-12} w^{2}, \quad Y_{\mathrm{A}}=14.3 \times 10^{-12} w^{2}$, and $l^{2}=L_{2}{ }^{2} / N_{\mathrm{A}} b_{\mathrm{A}}{ }^{2}=0.995$, by using eq 12,32 , and 36. On the other hand, when the $P(\theta)$ versus $X$ relation such as shown in Figure 8 is examined, it is found that the observed data can be well fitted by a calculated curve for an arc-shape model with $l^{2}=0.5$ : namely the observed anomalous curvature of the reciprocal $P(\theta)$ versus $X$ plot ( $c f$., Figure 8 ) can be explained by a model, in which the mean-square end-to-end distance 
of the central A-subchain is restricted about half of the unrestricted precursor homopolymer A-chain, presumably by the interaction between the two side B-subchains. However, the value of $\left\langle s^{2}\right\rangle_{\text {app }}$ calculated on this particular model (with $l^{2}=0.5$ ) is not in agreement with the observed value for the sample 27B: namely the former is estimated to be about $25-\%$ smaller than the value of the precursor PST $27 \mathrm{H}$; nevertheless, the latter is almost equal to that of the PST $27 \mathrm{H}$. On the other hand, the arc-shape model with the parameter $l^{2} \cong 1$ predicts a $P(\theta)$ versus $X$ relation that is almost identical to that of an unrestricted Gaussian chain: this of course fails to explain the anomalous curvature of the reciprocal $\boldsymbol{P}(\theta)$ versus $\boldsymbol{X}$ plot observed for the sample 27B ( $c f$., Figure 8). In addition, the value of $l^{2} \cong 1$ implies that the PST-subchain of the sample 27B has nearly the same dimensions as that of the precursor PST $27 \mathrm{H}$. The endto-end distance appears to be too large for the two PMMA-subchains to be able to undergo the intramolecular association, since the molecular weight of each PMMA-subchain is only about half of that of the PST-subchain. If the implication of $l^{2} \cong 1$ is true, then the block copolymer molecule would assume a conformation such as that represented by the model illustrated in Figure 1c. A calculation of the $P(\theta)$ function was also carried out for the model given in Figure 1c, i.e., the one consisting of an A-subchain of $N_{\mathrm{A}}$ segments having two free B-subchains, each consisting of $\left(N_{\mathrm{B}} / 2\right)$ segments. ${ }^{13}$ The results showed that the contribution from the B-subchains makes the reciprocal $P(\theta)$ to be a more slowly increasing function of $X$ than that of a free homopolymer chain: apparently this deviation is the reverse of the behavior of the observed plot.

Because of these controversies, the analysis of the PMMA-PST-PMMA triblock sample in $p \mathrm{XY}$ solution is still not conclusive. One possibility is that the sample $27 \mathrm{~B}$ assumes an arc-shape conformation with the central PSTsubchain having a restricted end-to-end distance, because of the intramolecular association (or attracting interaction) between the two side PMMA-subchains. In spite of this restriction, its $\left\langle s^{2}\right\rangle_{\text {app }}$ becomes large or, at least, comparable to that of the precursor PST $27 \mathrm{H}$, because of the intramolecular phase separation between the PST- and PMMA-subchains. There might of course be other possible explanations for the peculiar behavior of the PMMA-PST-PMMA triblock copolymers in $p \mathrm{XY}$ solution. However, at the moment, the authors believe that the arc-shape model, although qualitatively still inexact, provides a plausible explanation for all the peculiarities found so far for this system.

Acknowledgement. The authors wish to thank Dr. Hiroshi Ohnuma of Nagoya University for his indispensable contributions during the early stages of this study. They also wish to thank Dr. Teruo Fujimoto of Nagoya University for his valuable advice on the art of anionic polymerization.

\section{REFERENCES}

1. T. Kotaka, H. Ohnuma, and Y. Murakami, J. Phys. Chem., 70, 4099 (1966).

2. H. Ohnuma, T. Kotaka, and H. Inagaki, Polymer, 10, 501 (1969).

3. T. Kotaka, H. Ohnuma, and H. Inagaki, ibid., 10, 517 (1969).

4. H. Ohnuma, T. Kotaka, and H. Inagaki, Polymer J., 1, 716 (1970).

5. T. Kotaka, T. Tanaka, and H. Inagaki, ibid., 3, No. 4 (1972), in press.

6. P. Debye, J. Phys. Chem., 51, 18 (1947).

7. W. H. Stockmayer, L. D. Moore, Jr., M. Fixman, and B. N. Epstein. J. Polym. Sci., 16, 517 (1955).

8. W. Bushuk and H. Benoit, Can. J. Chem., 36, 1616 (1958).

9. M. Leng and H. Benoit, J. Polym. Sci., 57, 263 (1962).

10. K. Nagai, J. Chem. Phys., 38, 924 (1963).

11. P. J. Flory, "Statistical Mechanics of Chain Molecules,', Interscience Publishers, New York, N.Y., 1969, Chapters VIII and IX.

12. P. J. Flory and R. L. Jernigen, J. Amer. Chem. Soc., 90, 3128 (1968).

13. T. Tanaka, unpublished calculation.

14. H. Benoit, J. Polym. Sci., 11, 50 (1953).

15. Lord Rayleigh, Proc. Roy. Soc. (London), A84, 25 (1911).

16. M. Fixman, J. Chem. Phys., 23, 1656 (1955).

17. E. F. Casassa, J. Polym. Sci., Part A, 3, 605 (1965).

18. A. Horta, Macromolecules. 3, 371 (1970).

19. A. Peterlin, "Electromagnetic Scattering,' Pergamon Press, New York, N.Y., 1963, p 357. 
20. R. Koyama, J. Phys. Soc., Japan, 26, 493 (1966).

21. H. Inagaki, H. Suzuki, M. Fujii, and T. Matsuo, J. Phys. Chem., 70, 1718 (1966).

22. G. Dezelic, J. Chem. Phys., 45, 185 (1966).

23. B. H. Zimm, ibid., 16, 1099 (1948).

24. T. Fujimoto, private communication.

25. M. Morton, T. E. Helminiak, S. D. Gadkary, and F. Bueche, J. Polym. Sci., 57, 471 (1962).

26. G. C. Berry, paper presented at the IUPAC
Symposium on Macromolecular Chemistry, Tokyo-Kyoto, 1966; Preprints of Scientic Papers, 6, 144 (1966).

27. G. Grubisic, P. Rempp, and H. Benoit, $J$. Polym. Sci., Part B, 5, 753 (1967).

28. T. Fujimoto, H. Narukawa, and M. Nagasawa, Macromolecules, 3, 57 (1970).

29. I. Noda, T. Horikawa, T. Kato, T. Fujimoto, and M. Nagasawa, ibid., 3, 795 (1970). 